\title{
- Effect of Video Game Music on Hand Dexterity Performance in Young Adults
}

\author{
IJCRR \\ Section: Healthcare \\ ISI Impact Factor \\ (2019-20): 1.628 \\ IC Value (2019): 90.81 \\ $\operatorname{SJIF}(2020)=7.893$ \\ (c) (7) (8) \\ Copyright@IJCRR
}

\section{Hriday Shah ${ }^{1}$, Mugdha Oberoi ${ }^{2}$}

'Research Intern, K.J. Somaiya College of Physiotherapy, Sion, Mumbai, India; ${ }^{2}$ Assistant Professor, K.J. Somaiya College of Physiotherapy, Sion, Mumbai, India.

\section{ABSTRACT}

Introduction: Video game music genre has no lyrics, repeats indefinitely and has a 5-beat rhythm which acts as Rhythmic Auditory Stimuli. It can either be used as a stimulus providing rhythmic cues or as facilitating stimuli for training. It may activate various structures in the brain that help improve movement control and performance. Thus, the authors aimed to study the effects of video game music on hand dexterity performance.

Objectives: 1) To assess hand dexterity performance without music. 2) To assess hand dexterity performance with video game music. 3) To compare the hand dexterity performance with and without video game music.

Methods: In this cross over study design 230 asymptomatic individuals both male and female between the age groups 18 - 35 years were evaluated for hand dexterity performance using Bimanual turning test of Minnesota manual dexterity test. Randomly half of the subjects performed the test with video game music and after 24 hours re-performed the test without video game music, for the remaining half of the subjects the test conditions were reversed.

Results: The mean age of the population was 26.48 years where $50.9 \%$ were males and $49.1 \%$ females. The median time taken to finish the test with video game music was $138.07(89.98,597.29)$ seconds and without video game music was 133.73 (103.94, 216.61 ) seconds. The time taken to perform the test with video game music was $140.49+18.09$ seconds and without video game music was $142.28+47.84$ seconds. On comparing the two test conditions, the time taken to perform the test with video game music was less which was statistically significant with $p$-value $<0.0001$.

Conclusion: Hand dexterity performance with video game music is better as compared to without video game music.

Key Words: Chiptunes, Hand function, Motor performance, Rhythmic auditory stimuli, Bi-manual turning test

\section{INTRODUCTION}

Video game music is a soundtrack that accompanies the video game. This style of music is known as chiptunes, which combines simple melodic styles with more complex patterns or traditional music styles. Features of the video game music genre include a) music pieces designed to repeat indefinitely, that is a type of rhythmic auditory stimulus is present. b) The music pieces do not have lyrics hence act as auditory cues. c) A 5-beat rhythm is present that is used to create different effects. Video game music uses certain sound symbols helping the player to identify his goal in the task of the game he/she is playing and also focuses their perception on certain objects. ${ }^{1}$ Rhythmic Auditory Stimulation (RAS) is a neurological technique used for rehabilitation of rhythmic movements, intrinsically, and biologically. ${ }^{2}$

Hand dexterity includes hand and arm stability, reaction time, aiming, hand preference, finger tapping speed, wrist flexion speed and other different hand abilities and performance as the hand is the most involved, requited and participated part of the upper extremity. ${ }^{3}$ Authors of Oxford's Handbook for Music Education have also suggested the need for further research to know how video game music impacts physical activity performance. ${ }^{4}$

Video game music can be diegetic or non-diegetic depending on its arrangement during different stages of the game. This music helps in intriguing the player in the game due to its immersion effect, thus helping him improve his/her gaming performance. ${ }^{5}$

Corresponding Author:

Hriday Shah, Research Intern, KJ Somaiya College of Physiotherapy, Somaiya Ayurvihar Complex, Eastern Express Highway, Sion (E), Mumbai - 400022, Maharashtra, India; Phone: 02228125666; Mobile: 8879152114; E-mail: shah.hriday80@gmail.com

ISSN: $2231-2196$ (Print)

Received: 23.07 .2020
ISSN: 0975-5241 (Online)

Revised: 18.09 .2020
Accepted: 04.11 .2020

Published: 16.01 .2021 
Thus, the authors aimed to study the effect of video game music on hand dexterity performance in young adults. With the following objectives: 1) To assess hand dexterity performance without music. 2) To assess hand dexterity performance with video game music. 3) To compare the hand dexterity performance with and without video game music. Bimanual turning test of the Minnesota Hand dexterity test was used as an outcome measure.

\section{MATERIALS AND METHODS}

\section{Subjects}

This experimental cross over study included 230 asymptomatic young adults both male and female $(n=230)$ of the age group 18-35 years. Subjects with any musculoskeletal, neuromuscular, cardiovascular conditions and any individuals with impaired hearing were excluded. Written consent was obtained from all eligible subjects before enrollment.

\section{Intervention}

Approval was taken from the Ethics Committee, outward no. KJSCPT/886/18-19. The study was conducted at the research lab of K. J. Somaiya College of Physiotherapy. Subjects were randomly divided by the computer-generated selecting system into two sets. Set one, $(n=115)$ subjects first performed the hand dexterity test (Minnesota manual dexterity test: bimanual turning test) with video game music and after 24 hours re-performed the hand dexterity test without the video game music. Set two, $(n=115)$ subjects first performed the test without video game music and then after 24 hours re-performed the hand dexterity test with video game music.

\section{Outcome Measure}

The Bimanual Hand dexterity test of the Minnesota Manual Hand dexterity test was used as an outcome measure. A practice trial was given to the subject before the test. Then as shown in figure 1, the subjects were required to perform the test thrice and the final score calculated by the addition of all the 3 readings measured in seconds. The test conducted was the same only the study settings varied, which is with or without video game music.

\section{Procedure}

The objective of this test is to see how fast you can pick up the disks with one hand, turn them with the other hand, and replace the disks into the holes on the board. Subject with the LEFT hand, picks up the block from the upper righthand corner. Turns the disk while passing it to your RIGHT hand and return it into the original hole in the board with the BOTTOM side facing UP. Continue to until you complete the entire TOP row. As the subject starts the second row, say, with his/her RIGHT hand, picks up the first block in the second row. Turns the disk while passing it to LEFT hand and return it into the original hole with the BOTTOM side facing UP. The subject always picks UP the blocks with the hand that LEADS and put them DOWN with the hand that FOLLOWS. Test will be carried with/without video game music accordingly.

\section{Statistical Analysis}

To determine the difference between the time taken to perform the Bimanual Hand dexterity test with and without video game music, the scores of the test for each subject was recorded. The data collected did not pass the normality test and hence Wilcoxon signed-rank test was performed with $5 \%$ significance.

\section{RESULTS}

A total of 230 subjects participated in the study $(\mathrm{N}=230)$. The mean age of the population was 26.48 years where $50.9 \%$ were males and $49.1 \%$ females. The data collected did not pass the normality and Wilcoxon signed-rank test was used for analyses. On comparing the median values of the time taken to perform the test with video game music (89.98, 597.29) and without video game music $(103.94,216.61)$, the difference is bound to be statistically significant with p-value $<0.001$.

\begin{tabular}{|c|c|c|c|c|}
\hline & $\begin{array}{l}\text { With music } \\
\text { median } \\
\text { (range) } \\
\text { seconds }\end{array}$ & $\begin{array}{l}\text { Without } \\
\text { music me- } \\
\text { dian (range) } \\
\text { seconds }\end{array}$ & Test & $\begin{array}{c}\text { p- } \\
\text { value }\end{array}$ \\
\hline $\begin{array}{l}\text { Bimanual } \\
\text { Hand } \\
\text { dexterity } \\
\text { test }\end{array}$ & $\begin{array}{c}138.07(89.98 \\
597.29)\end{array}$ & $\begin{array}{c}133.73(103.94 \\
216.61)\end{array}$ & $\begin{array}{l}\text { Wilcoxon } \\
\text { signed } \\
\text { rank test }\end{array}$ & $<0.001$ \\
\hline
\end{tabular}

\section{DISCUSSION}

This study has assessed the effect of video game music on hand dexterity performance using the Minnesota Bimanual Hand Dexterity Test. It included young adults $(\mathrm{n}=230)$ both males $(n=117)$ and females $(n=113)$ within the range of 18 35 years of age (mean age $=26.48$ ). The hand dexterity performance was measured with and without video game music. Data analysis showed an extremely significant difference $(p<0.0001)$ in the median time taken to complete the Minnesota Bimanual Hand Dexterity Test with video game music $(89.98,597.29$ seconds) and without video game music (103.94, 216.61 seconds) conditions.

Lawrence assessed the players' performance of playing the video game, a motor function involving hand-eye co-ordination with background video game music of varying tempo, 
and the results obtained were not statistically significant. ${ }^{6}$ The study also explains that one of the reasons the effect of background music tempo may not affect the players' performance is because of practice due to the effect of non-declarative memory and procedural learning. Repetition of the same task improves the ability of the player to perform the task of playing the video game. But here, in the current study, every subject was their control group and hence the bias of learning effect would not be of much significance whether the test was performed first with or without video game music in the background.

The psychological experience of being involved in a video game is known as the immersion effect of the video game. The background music in video games has a tremendous effect on the players' gameplay by increasing the players' immersion while playing the video game because of the auditory feedback given to the brain due to different visual actions in the game giving a sense of real physical space. This research conducted by Zhang and Fu concluded that video games having background music attracted and appealed to many participants as compared to those video games that did not have any background music. ${ }^{5}$ Thus we can conclude from the above study that background music in the video game helps to improve the immersion, attention, and concentration of the subject which in-turn improves productivity by improving motor function.

Music therapy is an umbrella that includes many treatment techniques which incorporate paying attention and listening to music, singing notes and tunes with others and the therapist to improve language and speech articulation, playing musical instruments to improve functional hand movements, dexterity, and hand-eye co-ordination, playing piano and percussion, which improves gross and fine motor muscles using auditory and visual stimuli. ${ }^{2,7}$ Paul et al. in his studies described the therapeutic effects of music therapy on physical rehabilitation. Music therapy has shown to improve various physical and mental aspects such as strength, range of motion, balance, communication, and cognition. It also improves the quality of life in people with disabilities. ${ }^{8}$ Music therapy is growing in the field of rehabilitation. It has shown to improve varied patient conditions like Parkinsonism, Brain injuries and Psychological disorders, cardiac rehabilitation, etc. ${ }^{7,9}$

Rhythmic Auditory Stimulation (RAS) is one of the neurological techniques in music therapy which helps in the rehabilitation of rhythmic movements, intrinsically, and biologically. The physiological effects of auditory rhythm are the basis of RAS on motor control to improve movement rehabilitation of functional activities in neurological impairments. To achieve more functional gait patterns, immediate entertainment stimulus providing rhythmic cues during movement or as a facilitating stimulus are the two ways RAS can be used. ${ }^{2,9,10}$ According to some clinical reports, for the patient to achieve benefits while doing physical activities, rhythmic auditory stimuli was given while performing therapeutic motor exercises. ${ }^{11}$

RAS activates striatum of basal ganglia, cerebellum, and Reticulospinal connections by stimulating inferior colliculi, auditory projections, and audio-motor pathways. This stimulation reaches the supplementary motor area and premotor cortex helping in timing, sequencing, and response selection which improves motor function. ${ }^{17}$ Also, the reward-punishment centre of the brain stimulated by the limbic system is activated by music gives a sense of well - being. ${ }^{12}$ All these neurophysiological processes can be attributed to the improved performance to the hand dexterity test while video game music is on.

\section{CONCLUSION}

The hand dexterity performance with video game music was better as compared to without video game music. Video game music could potentially help to enhance motor performance. However, the results of the study should be valued for the mentioned population. Studying the properties of video game music in the future could help in establishing its place as an effective rhythmic auditory stimulus for improving motor performance. Video game music can be used as a Rhythmic Auditory Stimulus to improve motor function. The current authors studied the hand dexterity performance evaluated by Minnesota manual hand dexterity test and its effect because of video game music. The results indicated the time taken to perform the test with video game music was less which was statistically significant leading to the conclusion that the hand dexterity performance with the video game music was better as compared to without video game music.

\section{ACKNOWLEDGEMENT}

Authors acknowledge the immense help received from the scholars whose articles are cited and included in references to this manuscript. The authors are also grateful to authors/ editors/publishers of all those articles, journals and books from where the literature for this article has been reviewed and discussed.

\section{Financial support and scholarship - Nil}

Conflict of interest - There are no conflicts of interest.

\section{REFERENCES}

1. Collins K. Game sound. 1st ed. Cambridge, Mass.: MIT Press; 2008, Chapter 7: Gameplay, Genre and the functions of Game Audio, 123-137. 
2. Thaut MH. Rhythm, Music and the Brain, New York and London: Taylor and Francis Group, Neurologic Music Therapy Techniques and Definitions, Roultedge Publications 2005;1:112.

3. Martin JA, Ramsay J, Hughes C, Peters DM, Edwards MG, Age and Grip Strength Predict Hand Dexterity in Adults. PLoS One 2015;10(2):

4. Welch G, McPherson G. The Oxford handbook of music education. Volume 2. Oxford: Oxford University Press. Let's Learn: Let's Play, 2012;554

5. Zhang $\mathrm{J}, \mathrm{Fu} \mathrm{X}$, The Influence of Background Music of Video Games on Immersion. J Psychoth 2015;05(04).

6. Lawrence D. The effect of musical tempo on video game performance [Post graduation]. Uni Jyvaskyla 2012; 3(5): 234-239.

7. Jang-won L, Yong KK, Jung HC, Soyoung L. The Effectiveness of Music Therapy on Cerebral Palsy Patients Receiving Reha- bilitation Treatment, Int J Humanit Soc Sci Invent 2016; 5(9): 24-29.

8. Paul S, Ramsey D. Music therapy in physical medicine and rehabilitation. Aus Occup Ther J 2000;47(3):111-118.

9. Thaut MH, McIntosh GC, Hoemberg V. Neurobiological foundations of neurologic music therapy: rhythmic entrainment and the motor system. Front Psychol 2015;5:1185.

10. Thaut M, Schleiffers S, Davis W. Analysis of EMG Activity in Biceps and Triceps Muscle in an Upper Extremity Gross Motor Task under the Influence of Auditory Rhythm. J Music Ther 1991;28(2):64-88

11. Sobana R, Sundar S, Jaiganesh K. Music Therapy for Pulmonary Rehabilitation in Chronic Obstructive Pulmonary Disease [COPD] Patients- An Interventional Trial. Int J Curr Res Rev 2020;12(12):26-29.

\section{GRAPHICAL ABSTRACT}

\section{Effect of Video Game Music On Hand Dexterity Performance in Young Adults}

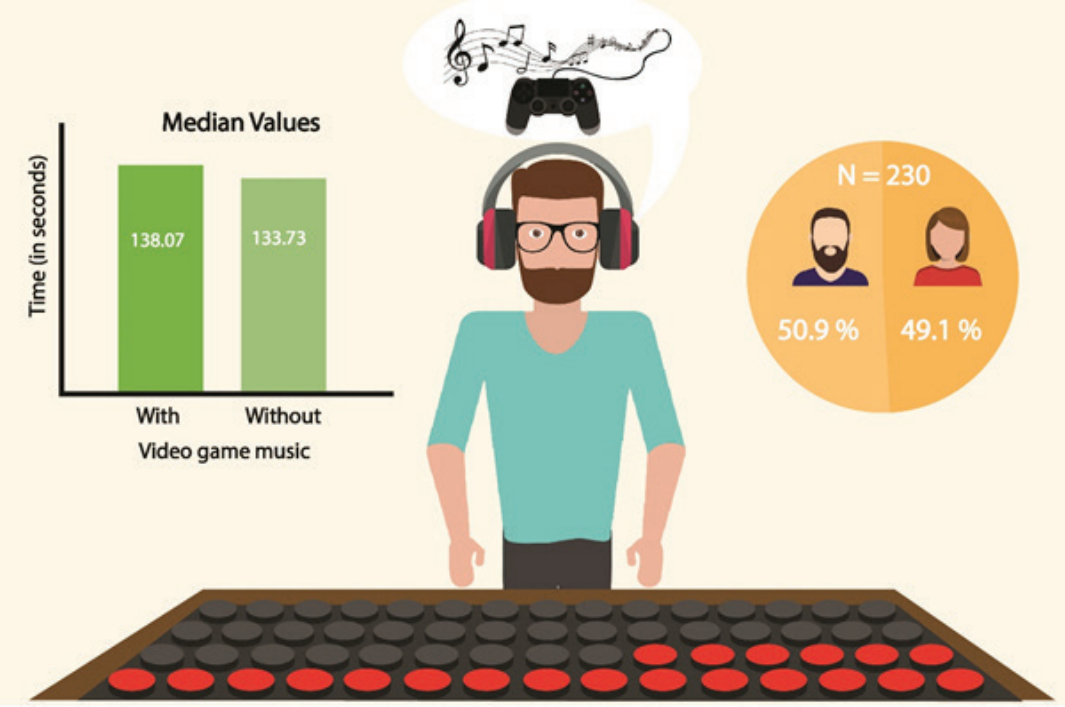

